Supporting Information

\title{
Selective Substrate-Orbital-Filtering Effect to Realize Large-Gap Quantum Spin Hall Effect
}

Huisheng Zhang, ${ }^{\dagger}$ Yingying Wang, ${ }^{\dagger}$ Wenjia Yang, ${ }^{\dagger}$ Jingjing Zhang, ${ }^{\dagger}$ Xiaohong Xu, ${ }^{*}, \dagger$ and Feng Liu*,

Key Laboratory of Magnetic Molecules and Magnetic Information Materials of Ministry of Education \& Research Institute of Materials Science of Shanxi Normal University , Linfen, 041004, China

Department of Materials Science and Engineering, University of Utah, Salt Lake City, Utah 84112, United States

*E-mail: xuxh@sxnu.edu.cn; fliu@eng.utah.edu

\section{Details of First-principles calculations}

We have performed first-principles calculations of structural, electronic, and topological properties of pristine and $\mathrm{BaTe}(111)$ surface-supported plumbene by using the projector augmented wave (PAW) formalism [1] in the Vienna ab initio simulation package (VASP) [2]. The Perdew-Burke-Ernzerhof (PBE) approximation was used to describe the exchange and correlation functional [3]. The lattice constants of pristine planar and low-buckled plumbene were fully optimized. For the $\mathrm{BaTe}(111)$ surface-supported plumbene, we adopted the experimental lattice constant of $4.95 \AA$ [4]. The BaTe(111) substrate was modeled with a slab of five atomic layers, where the atoms in the lowest two layers are fixed in their bulk positions. The bottom layer of Te atoms was terminated by hydrogen atoms,. A vacuum space of larger than $20 \AA$ was used to avoid interaction between two adjacent supercells. The plane-wave cutoff 
energy was set to $500 \mathrm{eV}$ and all the atoms in the supercells were allowed to move until the Hellmann-Feynman force on each atom is smaller than $0.01 \mathrm{eV} / \AA$. The $\Gamma$-centered Monkhorst-Pack $k$-point mesh of $18 \times 18 \times 1$ was used in all calculations. The van der Waals (vdW) correction with the Grimme (DFT-D2) method [5] was included in the substrate-supported plumbene calculations. The $\mathrm{Z}_{2}$ invariant, Wannier Charge Center (WCC) and edge states were calculated to identify the topological properties of pristine and substrate-supported plumbene by using Wannier90 [6] and WannierTools [7].

\section{Three-orbitals TB model}

We employed the tight-binding (TB) model to explore the topological mechanism induced by $p_{x, y}-p_{z}$ band inversion in $\mathrm{Pb}-\mathrm{BaTe}(111)$. The TB model was constructed for a honeycomb lattice with two $p_{x, y}$ orbitals on A site and one $p_{z}$ orbital on $\mathrm{B}$ site (Figure $2 \mathrm{a}$ ), respectively, the Hamiltonial $H(k)$ with a $3 \times 3$ matrix was written as

$$
H_{\text {hop }}(k)=\sum_{\alpha} \varepsilon_{\alpha} c_{0 \alpha}^{+} c_{0 \alpha}+\sum_{i} \sum_{\alpha, \beta}\left(t_{0 \alpha, i \beta} c_{0 \alpha}^{+} c_{i \beta}+t_{i \beta} c_{i \beta}^{+} c_{0 \alpha}\right),
$$

where $\alpha, \beta=p_{x}, p_{y}, p_{z}$ are the orbital index, $\varepsilon_{\alpha}$ is the on-site energy and $t_{0 \alpha, i \beta}$ is the nearest-neighbor $(\mathrm{NN})$ hopping parameter. $c^{+}$and $c$ are creation and annihilation operators. The $3 \times 3$ matrix elements without considering spin-orbit coupling (SOC) are expressed as

$$
\begin{aligned}
& H_{11}=\varepsilon_{p x}+2 \cos \sqrt{3} k_{y} V_{p p \pi}+\left(\frac{3}{2} V_{p p \sigma}+\frac{1}{2} V_{p p \pi}\right) \times\left[\cos \left(\frac{3}{2} k_{x}+\frac{\sqrt{3}}{2} k_{y}\right)+\cos \left(\frac{3}{2} k_{x}-\frac{\sqrt{3}}{2} k_{y}\right)\right], \\
& H_{12}=\frac{\sqrt{3}}{2}\left(V_{p p \sigma}-V_{p p \pi}\right) \times\left[\cos \left(\frac{3}{2} k_{x}+\frac{\sqrt{3}}{2} k_{y}\right)-\cos \left(\frac{3}{2} k_{x}-\frac{\sqrt{3}}{2} k_{y}\right)\right], \\
& H_{13}=\frac{1}{2}\left(V_{p p \sigma}-V_{p p \pi}\right) \times\left[e^{i k_{x}}-e^{-\frac{i}{2} k_{x}} \times \cos \frac{\sqrt{3}}{2} k_{y}\right], \\
& H_{22}=\varepsilon_{p y}+2 \cos \sqrt{3} k_{y} V_{p p \sigma}+\left(\frac{1}{2} V_{p p \sigma}+\frac{3}{2} V_{p p \pi}\right) \times\left[\cos \left(\frac{3}{2} k_{x}+\frac{\sqrt{3}}{2} k_{y}\right)+\cos \left(\frac{3}{2} k_{x}-\frac{\sqrt{3}}{2} k_{y}\right)\right], \\
& H_{23}=\frac{\sqrt{3}}{2} i\left(V_{p p \sigma}-V_{p p \pi}\right) \times e^{-\frac{i}{2} k_{x}} \times \sin \frac{\sqrt{3}}{2} k_{y}, \\
& H_{33}=\varepsilon_{p z}+2 \cos \sqrt{3} k_{y} V_{p p \pi}+2 V_{p p \pi} \times\left[\cos \left(\frac{3}{2} k_{x}+\frac{\sqrt{3}}{2} k_{y}\right)+\cos \left(\frac{3}{2} k_{x}-\frac{\sqrt{3}}{2} k_{y}\right)\right], \\
& H_{21}=H_{12}^{*}, H_{32}=H_{23}^{*}, H_{31}=H_{13}^{*} .
\end{aligned}
$$


where $\varepsilon_{p x}, \varepsilon_{p y}$, and $\varepsilon_{p z}$ are on-site energies of $p_{x}, p_{y}$, and $p_{z}$ orbitals, respectively. $V_{p p \sigma}$ and $V_{p p \pi}$ are NN hopping parameters. The on-site SOC term is added as

$$
H_{S O C \uparrow / \downarrow}=s \cdot \lambda_{S O C}\left[\begin{array}{ccc}
0 & -i & 0 \\
i & 0 & 0 \\
0 & 0 & 0
\end{array}\right],
$$

where $\lambda_{S O C}$ is the atomic SOC strength, and $s= \pm 1$ represent spin-up and spin-down channels, respectively. Here, the parameters are set to $\varepsilon_{p x}=\varepsilon_{p y}=-1.1 \mathrm{eV}$, $\varepsilon_{p z}=1.1 \mathrm{eV}, V_{p p \pi}=-0.1 \mathrm{eV}, V_{p p \sigma}=0.6 \mathrm{eV}$. $\lambda_{S O C}$ is set to 0 and $0.5 \mathrm{eV}$ in Figure $3 \mathrm{a}$ and $4 \mathrm{~b}$, respectively.

\section{Electronic and topological properties of $\mathrm{PbX}-\mathrm{BaTe}(111)$}

Similar to hydrogenated $\mathrm{Pb}-\mathrm{BaTe}(111), \mathrm{BaTe}(111)$-supported plumbene are also halogenated, labeled as $\mathrm{PbX}-\mathrm{BaTe}(111)(\mathrm{X}=\mathrm{F}, \mathrm{Cl}, \mathrm{Br}$, or I). It is expected that $\mathrm{PbX}-\mathrm{BaTe}(111)$ hosts large-gap QSH state. Figure S2a-d gives the corresponding band structures without SOC, where each system exhibits semiconducting state and two quadratic bands are degenerate at the Fermi level. By considering SOC, a large bandgap varying from $0.45 \mathrm{eV}$ to $0.55 \mathrm{eV}$ is obtained, as shown in Figure $2 \mathrm{f}-\mathrm{h}$. The calculated nontrivial $Z_{2}=1$ is obtained for each system, demonstrating that all halogenated $\mathrm{Pb}-\mathrm{BaTe}(111)$ systems are large-gap QSH insulators. 


\section{Supplementary Figures and captions}

(a)

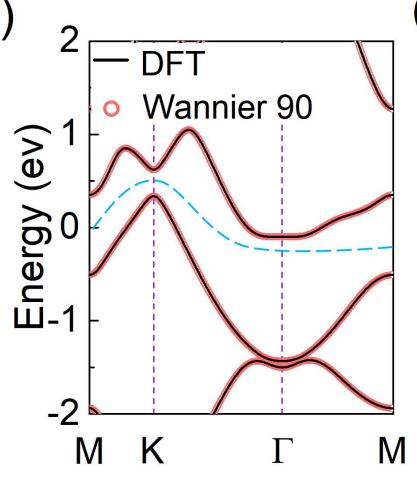

(c)

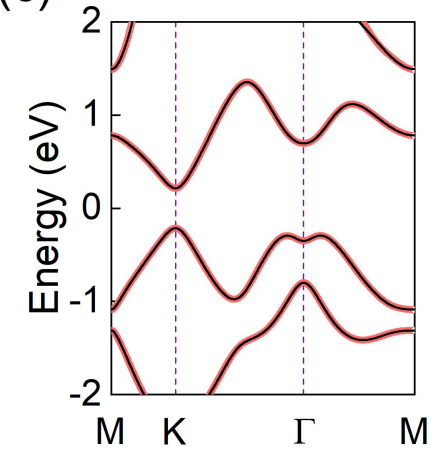

(b)

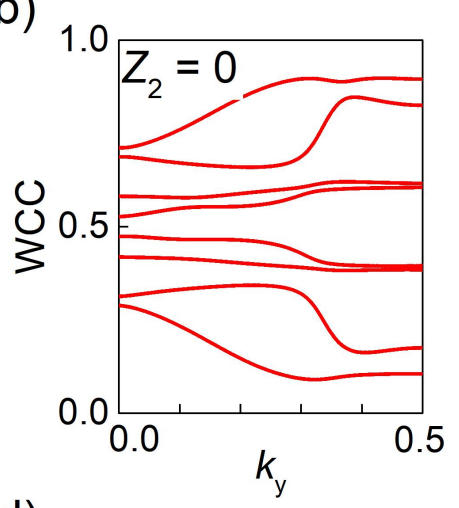

(d)

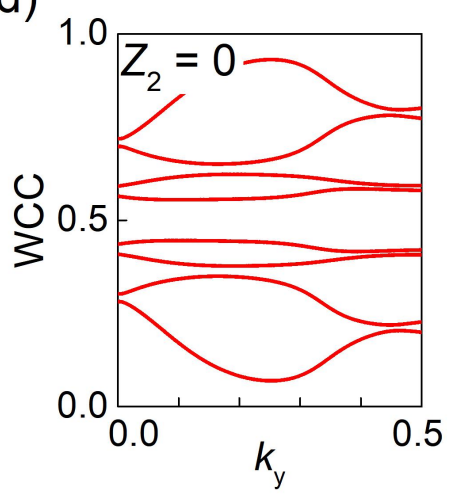

Figure S1. (a) Calculated band structures of planar plumbene with SOC using DFT method (solid black curves) and Wannier interpretation (open orange circles). The blue dashed line denotes "curved" Fermi level. (b) The corresponding Wannier charge center (WCC) and calculated $Z_{2}$ invariant. (c,d) The same as $(\mathrm{a}, \mathrm{b})$ but for low-buckled plumbene. 

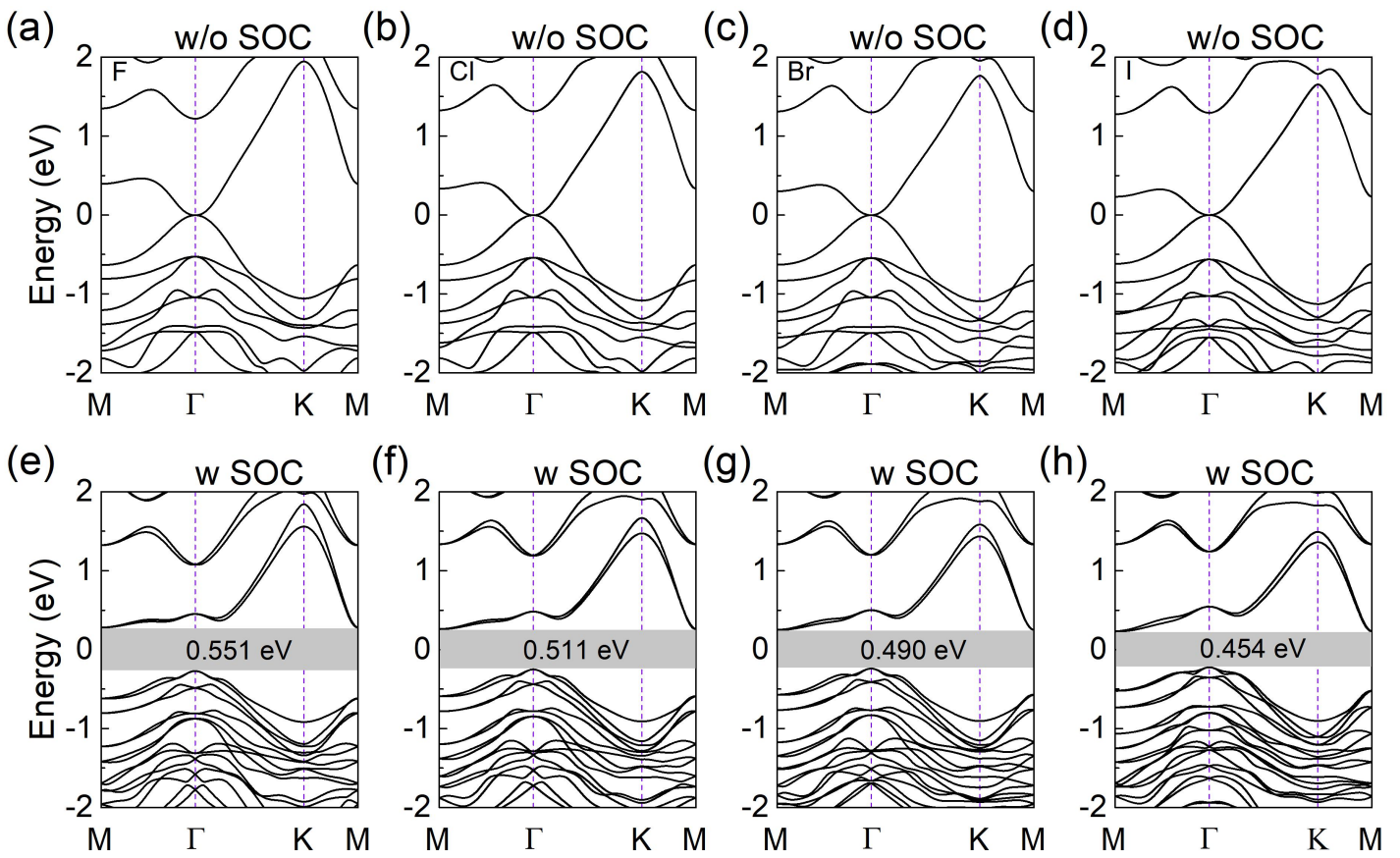

Figure S2. The band structures of PbF-BaTe(111), PbCl-BaTe(111), $\mathrm{PbBr}-\mathrm{BaTe}(111)$, and $\mathrm{PbI}-\mathrm{BaTe}(111)$ without (a-d) and with (e-f) SOC, respectively. The calculated $Z_{2}$ invariant is 1 for each system with SOC. 
(a)
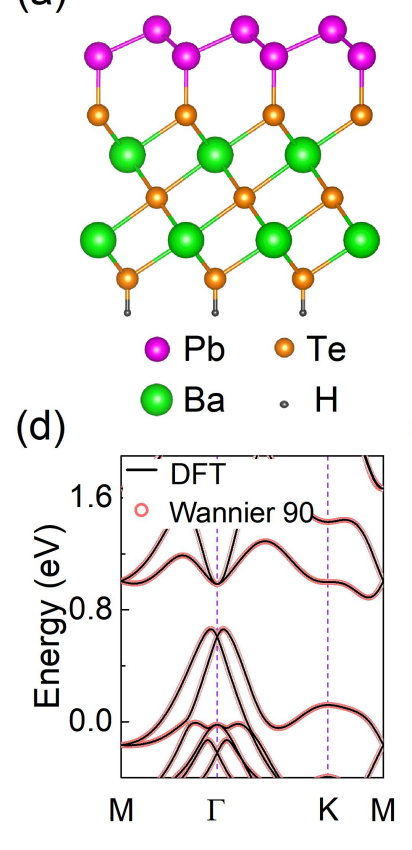

(b)

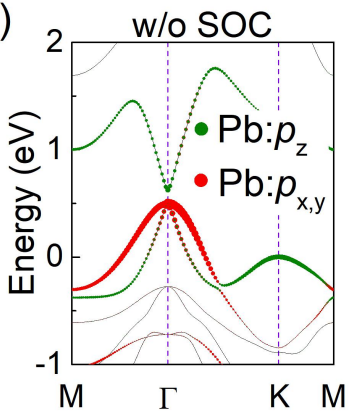

(e)

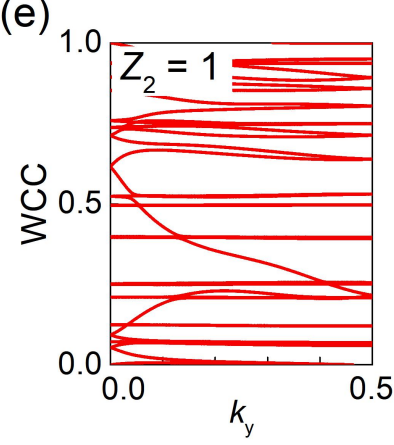

(c)

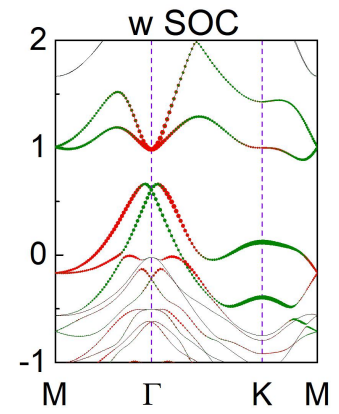

(f)

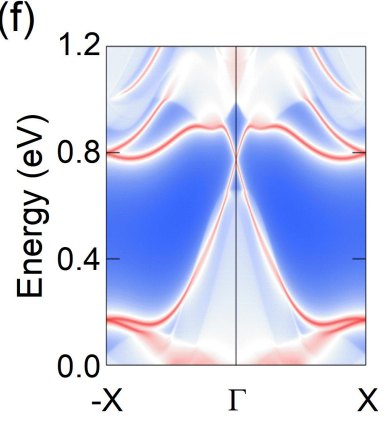

Figure S3. (a) Side view of the $3 \times 3$ supercell for another configuration of $\mathrm{Pb}-\mathrm{BaTe}(111)$. The corresponding band structures without (b) and with (c) SOC, respectively. The projected bands of $\mathrm{Pb} p_{x, y}$ and $p_{z}$ orbitals are also shown. (d) Calculated band structures of $\mathrm{Pb} / \mathrm{BaTe}(111)$ with SOC using DFT method (solid black curves) and Wannier interpretation (open orange circles). (e) The corresponding WCC and $Z_{2}$ invariant. (f) Edge states of $\mathrm{Pb} / \mathrm{BaTe}(111)$. 

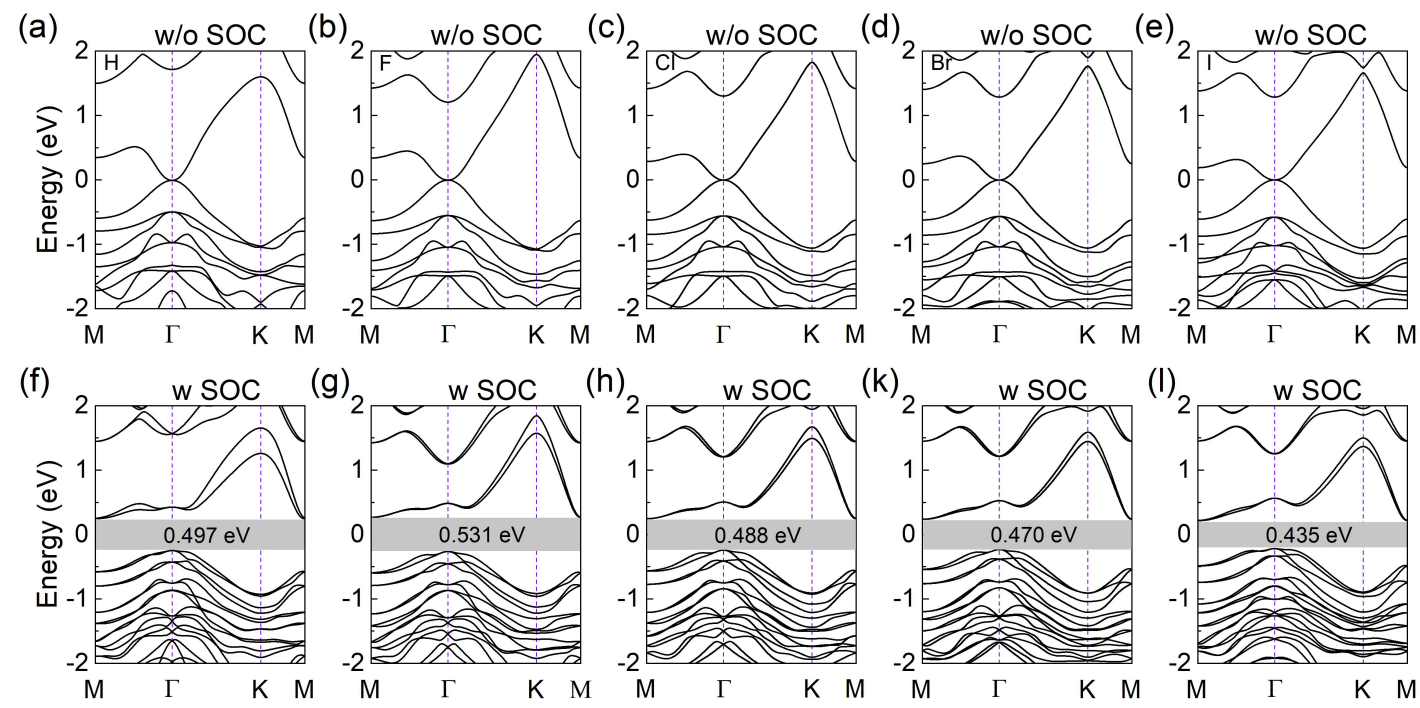

Figure S4. The band structures for another configuration (Figure S3a) of $\mathrm{PbH}-\mathrm{BaTe}(111)$, PbF-BaTe(111), PbCl-BaTe(111), PbBr-BaTe(111), and PbI-BaTe(111) without (a-e) and with (f-1) SOC, respectively. The calculated $Z_{2}$ invariant is 1 for each system with SOC. 
(a)

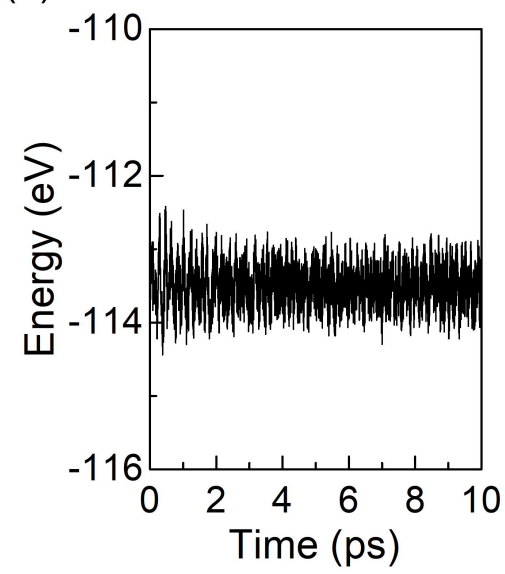

(b)

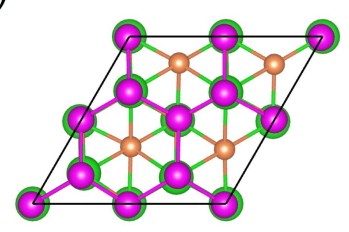

(c)

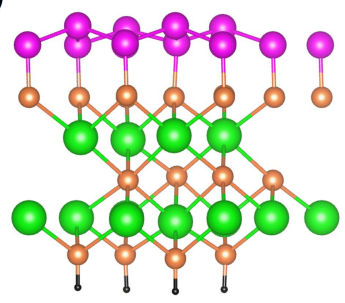

Figure S5. (a) Ab initio molecular dynamics simulations of Pb-BaTe(111) at $300 \mathrm{~K}$. (b) and (c) display the final structure after $10 \mathrm{ps}$, showing that its ordered structure is well preserved. 


\section{Reference}

1 Kresse, G.; Joubert, D. From Ultrasoft Pseudopotentials to the Projector Augmented-Wave Method. Phys. Rev. B: Condens. Matter Mater. Phys. 1999, 59, 1758-1775.

2 Kresse, G.; Furthmüller, J. Efficient Iterative Schemes for Ab Initio Total-Energy Calculations Using a Plane-Wave Basis set. Phys. Rev. B: Condens. Matter Mater. Phys. 1996, 54, 11169-11186.

3 Perdew, J. P.; Burke, K.; Ernzerhof, M.; Generalized Gradient Approximation Made Simple. Phys. Rev. Lett. 1996, 77, 3865-3868.

4 Partin, D. L.; Thrush, C. M.; Clemens, B. M. Lead Strontium Telluride and Lead Barium Telluride Grown by Molecular-Beam Epitaxy. J. Vac. Sci. Technol. B. 1987, 5, 686-689.

5 Grimme, S. Semiempirical GGA-Type Density Functional Constructed with a Long-Range Dispersion Correction. J. Comput. Chem. 2006, 27, 1787-1799.

6 Mostofi, A. A.; Yates, J. R.; Lee, Y. S.; Souza, I.; Vanderbilt, D.; Marzari, N. Wannier90: A Tool for Obtaining Maximally-Localised Wannier Functions. Comput. Phys. Commun. 2008, 178, 685-699.

7 Wu, Q.; Zhang, S.; Song, H. F.; Troyer, M.; Soluyanov, A. A. WannierTools: An Open-Source Software Package for Novel Topological Materials. Comput. Phys. Commun. 2018, 224, 405-416. 